\title{
Enhanced Rate Adaptation Schemes with Collision Awareness ${ }^{\star}$
}

\author{
Seongkwan Kim ${ }^{1}$, Sunghyun Choi $^{1}$, Daji Qiao ${ }^{2}$, and Jongseok Kim ${ }^{3}$ \\ ${ }^{1}$ School of Electrical Engineering and INMC, Seoul National University, Korea \\ 2 Department of Electrical and Computer Engineering, Iowa State University, USA \\ ${ }^{3}$ National Defense and Communications Institution, Korea \\ skim@mwnl.snu.ac.kr, schoi@snu.ac.kr, \\ daji@iastate.edu, sigsch7820@army.mil.kr
}

\begin{abstract}
While many existing rate adaptation schemes in IEEE 802.11 Wireless LANs result in severe throughput degradation since they do not consider the collision effect when selecting the transmission rate, CARA (Collision-Aware Rate Adaptation) [1] shows improved system performance thanks to its collision-awareness capability. In this paper, we propose two enhancements to the original CARA scheme to further improve the system performance. The first one is called $C A R A-R I$, which extends CARA's collision-awareness capability in making rate increase decisions, while the second one, called $C A R A-H D$, incorporates a hidden station detection mechanism. Simulation results show that the proposed schemes outperform the original CARA significantly under various randomly-generated network topologies.
\end{abstract}

\section{Introduction}

While the 802.11 PHYs (Physical layers) provide multiple transmission rates, the IEEE 802.11 standard [2] does not specify any algorithm to utilize the multiple transmission rates efficiently. Over the years, many rate adaptation schemes have been proposed in the literature and in practical devices.

In a typical 802.11 system, multiple stations contend for the shared wireless medium; frame collisions are inevitable due to the contention nature of the 802.11 DCF (Distributed Coordination Function). Therefore, the effectiveness of a rate adaptation scheme depends on not only how fast it can respond to the variation of wireless channel, but more importantly, how frame collisions may be detected and handled properly.

There are two major categories of rate adaptation schemes: closed-loop and open-loop schemes. Closed-loop schemes rely on the interaction between transmitter and receiver, and the rate adaptation is dictated by the receiver. In comparison, open-loop schemes, with which a station makes the rate adaptation decision solely based on its local Ack (Acknowledgment) information, are

\footnotetext{
* This research was in part supported by Information Technology Research Center (ITRC) and Samsung Advanced Institute of Technology (SAIT).
} 
generally standard-compliant and very simple to implement, and hence, more popular. The most widely-adopted ARF (Automatic Rate Fallback) scheme 3] is an open-loop scheme.

However, most existing open-loop schemes malfunction severely when there are many contending stations in the network, because they can not differentiate frame collisions from frame transmission failures caused by channel errors, and hence, may decrease the transmission rate over-aggressively. To address such problem, we proposed a novel rate adaptation scheme with collision-awareness capability, called CARA (Collision Aware Rate Adaptation) in [1. CARA combines adaptive RTS/CTS (Request-to-Send/Clear-to-Send) exchange with CCA (Clear Channel Assessment) functionality to detect frame collisions. As a result, compared with other open-loop rate adaptation schemes, CARA is more likely to make the correct rate adaptation decisions, and hence, performs better.

In this paper, we propose two enhancements to the original CARA to further improve the system performance. The first one is called $C A R A-R I$ (Rate Increase), which extends CARA's collision-awareness capability in making rate increase decisions, while the second one, called $C A R A-H D$ (Hidden Detection), incorporates a hidden station detection mechanism.

\section{Proposed Schemes}

In this section, we describe the details of CARA-RI and CARA-HD. We use $C A R A-B A S I C$ to refer to the original CARA schemen, in order to differentiate it from the proposed extensions.

\subsection{CARA-RI (CARA-Rate Increase)}

CARA-BASIC yields higher throughput than ARF by making collision-aware rate decrease decisions. However, when attempting to increase the transmission rate, it simply employs ARF's counting algorithm and resets the consecutive success count $(m)$ upon any frame transmission failure. The key idea of CARA-RI is to introduce collision-aware rate increase decisions into CARA-BASIC. Specifically, unlike in ARF and CARA-BASIC, where $m$ is reset with any frame loss, CARA-RI only resets $m$ when the frame loss occurs with RTS/CTS preceding the transmission attempt. In other words, $m$ is reset only when the frame loss can be clearly identified as a channel-error-caused failure. As a result, CARA-RI adjusts the transmission rate more quickly to the improving wireless channel condition than CARA-BASIC.

\subsection{CARA-HD (CARA-Hidden Detection)}

In the presence of hidden stations in a WLAN, the performance of the basic DCF can be severely degraded. It is because of the drastically increasing collision

${ }^{1}$ The detailed description of CARA and discussions on its effectiveness are omitted due to space limitation. Refer to [1] for more information. 
probability caused by hidden stations. The unprotected time interval, however, can be shortened to the RTS transmission time, by preceding the data frame transmission with the exchange of two short control frames, i.e., RTS and CTS frames, and hence, the hidden station problem can be ameliorated.

Thanks to its collision awareness capability, CARA-BASIC achieves better performance than ARF in a hidden station environment. However, as pointed out in 4, CARA-BASIC might suffer from RTS oscillation, which alternates in turning on and off the RTS frame transmission.

To better deal with the hidden station problem, we propose the second extension, called CARA-HD, which incorporates a hidden station detection mechanism that we proposed recently in 5 . The hidden station detection mechanism works as follows. We assume an infrastructure-based WLAN system, where all stations can hear the AP, but stations may be hidden to each other. Let $A$ and $B$ denote a pair of hidden stations in the network. Although $A$ cannot hear the data transmission from $B$ to the AP, it can hear at least the corresponding Ack frame transmission from the AP to $B$. By looking into either the frame content or the frame length information carried in the physical layer header, $A$ should be able to figure out that this is an Ack frame transmission, and consequently, if $A$ did not hear any data frame transmission associated with this Ack, it can claim detection of a hidden station (in this example, station $B$ ).

\section{Performance Evaluation}

In this section, we evaluate the following schemes in randomly-generated network topologies by using the $n s$-2 simulator: (1) ARF; (2) CARA-BASIC; (3) CARARI; (4) CARA-HD; and (5) CARA-HYBRID, which combines CARA-RI and CARA-HD. We simulate an infrastructure-based 802.11 b system with the same simulation setup as that used in [1. We set the operational parameters, such as the consecutive success threshold $\left(M_{t h}\right)$, the consecutive failure threshold $\left(N_{t h}\right)$, and the probe activation threshold $\left(P_{t h}\right)$ as those used in [1].

In a random-topology network, all the stations are randomly placed within a circle around the AP with the radius of 80 meters. The transmission range of a station is set to 80 meters, and hence, hidden stations are likely to exist in the network. We assume a time-varying Ricean fading channel model with Ricean factor $K=3 \mathrm{~dB}$ to describe the indoor fading channel. We vary the number of contending stations from 5 to 20. Simulation results are plotted in Fig. 1] where each point is averaged over 50 simulation runs.

In general, CARA schemes are significantly better than ARF in terms of aggregate system throughput, thanks to their collision-awareness capabilities. CARA-HD always performs better than CARA-BASIC, and as the number of contending stations increases, the performance gap between them becomes more significant. This is because, in a randomly-generated network, with more contending stations, there is a higher probability that hidden stations exist in the network, and, under such circumstances, CARA-HD becomes more effective. 


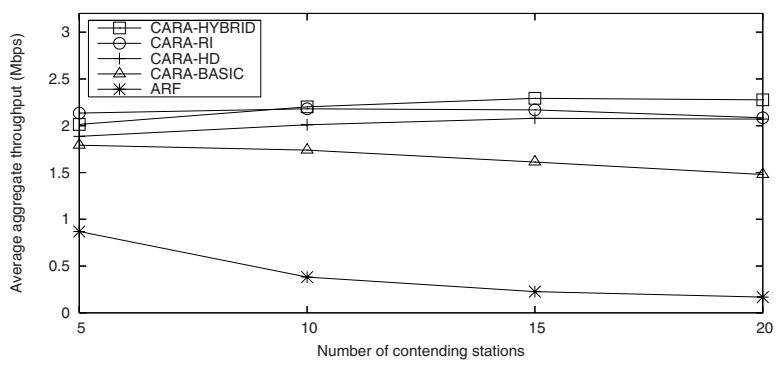

Fig. 1. Throughput comparison in random-topology networks

It is interesting to see that CARA-RI indeed yields higher aggregate throughput than all other testing schemes except CARA-HYBRID. This is because CARA-RI increases the transmission rate more proactively. As a result, data frames are generally transmitted at higher rates with CARA-RI, and hence, time wasted on collisions and retransmissions is shorter. However, as the number of contending stations increases and, in turn, the probability that hidden stations exist increases, the effect of the proactive rate increase is comparable with that of the hidden detection mechanism. This is evidenced by the similar performances of CARA-RI and CARA-HD shown in Fig. 11 when there are 20 contending stations in the network.

CARA-HYBRID conducts both collision-aware rate increases and hidden station detection. From the figure, we observe that, under certain scenarios, CARAHYBRID performs worse than CARA-RI. This is surprising at the first sight but rather reasonable for the following reason. Recall that CARA-HD deals with hidden station problem and reduces the collision length by enforcing an RTS/CTS exchange before each data transmission attempt. So when the extra RTS/CTS exchange overhead is larger than the overhead caused by collisions and retransmissions, combining CARA-HD with CARA-RI indeed degrades the throughput performance.

\section{References}

1. Kim, J., et al.: CARA: Collision-Aware Rate Adaptation for IEEE 802.11 WLANs. In: Proc. IEEE INFOCOM. (2006)

2. IEEE 802.11-1999, Part 11: Wireless LAN Medium Access Control (MAC) and Physical Layer (PHY) specifications. (August 1999)

3. Kamerman, A., et al.: WaveLAN-II: a high-performance Wireless LAN for the Unlicensed Band. Bell Labs Technical Journal 2(3) (1997)

4. Wong, S., et al.: Robust Rate Adaptation for 802.11 Wireless Networks. In: Proc. ACM MobiCom. (2006)

5. Kim, Y., et al.: A Novel Hidden Station Detection Mechanism in IEEE 802.11 WLAN. IEEE Commun. Lett. 10(8) (2006) 